\title{
Craig Interpolation for the Integers: Results, Implementation, and Experiences
}

\author{
Philipp Rümmer \\ Department of Information Technology, Uppsala University, Sweden
}

\begin{abstract}
Craig interpolation is a versatile tool in formal verification, in particular for generating intermediate assertions in safety analysis and model checking. Over the last years, a variety of interpolation procedures for linear integer arithmetic (and extensions) have been developed. I will give an overview of the existing algorithms and design choices, and then discuss implementations of such procedures within theorem provers and SMT solvers. In particular, I will describe an implementation done using the multi-paradigm language Scala, which is built on top of the Java runtime infrastructure, and evaluate performance and engineering aspects.
\end{abstract}

